\title{
Relation between exposure related indices and neurological and neurophysiological effects in workers previously exposed to mercury vapour
}

\author{
Dag G Ellingsen, Tore Mørland, Arne Andersen, Helge Kjuus
}

\begin{abstract}
A cross sectional study of aspects of their neurology was carried out on 77 chloralkali workers previously exposed to mercury $(\mathrm{Hg})$ vapour and compared with 53 age matched referents. The chloralkali workers had been exposed for an average of 7.9 years at a concentration of $59 \mu \mathrm{g} \mathrm{Hg} / \mathrm{m}^{3}$ in the working atmosphere. The individual mean urinary concentration of $\mathrm{Hg}$ for each year of exposure was $531 \mathrm{nmol} \mathrm{Hg} / 1$. On average the exposure had ceased 12.3 years before the examinations. Both the median sensory nerve conduction velocity and the amplitude of the sural nerve were associated with measures of cumulative exposure to $\mathrm{Hg}$. An association was also found between years since first exposure to $\mathrm{Hg}$ and aspects of the visual evoked response. Previously exposed subjects with postural tremor or impaired coordination also had alterations in visual evoked response. These results may indicate an effect of previous exposure to mercury vapour on the nervous system, possibly in the visual pathway, cerebellum, and the peripheral sensory nerves.
\end{abstract}

(British Fournal of Industrial Medicine 1993;50:736-744)

Inhaled mercury $(\mathrm{Hg})$ vapour is absorbed through the alveolar membrane into the blood where it is oxidised to divalent $\mathrm{Hg}$. Elementary $\mathrm{Hg}$ in the blood can pass the blood brain barrier by diffusion. Inorganic $\mathrm{Hg}$ is mainly excreted into urine and faeces. ${ }^{1}$

Department of Occupational Medicine, Telemark Central Hospital, N-3906 Porsgrunn, Norway

D G Ellingsen, $\mathrm{H}$ Kjuus

Department of Neurology, Telemark Central

Hospital, N-3710 Skien, Norway

T Mørland, A Andersen
Ongoing exposure to $\mathrm{Hg}$ vapour has been associated with a wide range of symptoms from the nervous system and numerous investigations have been performed to study neurological signs and neurophysiological abnormalities. ${ }^{2-16}$ Few studies have focused on long term neurological abnormalities after ceasing exposure to $\mathrm{Hg}$ vapour. One study showed an increased prevalence of neurological abnormalities such as impaired coordination, tremor, and decreased sensation among subjects for whom exposure had ceased more than 20 years before examination. ${ }^{17}$ The prevalence of finger tremor and disturbance of fine movements diagnosed in $\mathrm{Hg}$ miners at the time of $\mathrm{Hg}$ intoxication was reduced at the follow up, which was from two months to 17 years later. ${ }^{18}$

A post mortem study of two former $\mathrm{Hg}$ miners more than 10 years after stopping exposure showed the highest $\mathrm{Hg}$ concentrations in the occipital lobe and substantia nigra. ${ }^{19} \mathrm{~A}$ thermometer filler exposed to $\mathrm{Hg}$ vapour for 18 months had a raised $\mathrm{Hg}$ content in his brain 16 years after exposure had ceased. ${ }^{20}$ In a study by Kosta et al, ${ }^{21}$ brain $\mathrm{Hg}$ concentrations among retired $\mathrm{Hg}$ miners were shown to be roughly 100 -fold higher than in unexposed referents.

Evaporation of liquid $\mathrm{Hg}$ used as a cathode in the electrolysis of brine in the production of chlorine and caustic soda may result in a health hazard to the workers in the chloralkali industry.

During 1989, 77 workers previously exposed to $\mathrm{Hg}$ vapour at a chloralkali plant which started production in 1947 and was closed in 1987 were studied by neurological examination, neurography, and evoked responses. The main results from that study were an increased prevalence of postural tremor, impaired coordination, and reduced distal sensation among the exposed subjects. Prolongation of N75 in the visual evoked response and reduced median sensory and motor nerve conduction velocities were found among the subjects with the highest exposure. ${ }^{22}$

The purpose of the present investigation was to further study the relation between different indices 
of $\mathrm{Hg}$ exposure and neurological and neurophysiological results among subjects with past exposure to $\mathrm{Hg}$ vapour.

\section{Materials and methods}

\section{STUDY DESIGN}

The inclusion criteria for the study were that the workers should be men under 65 years of age exposed to $\mathrm{Hg}$ vapour for more than one year between 1947 and 1987. Individual measurements of urinary $\mathrm{Hg}$ from at least four different three monthly periods during their time of exposure were also required. These criteria were met by $130 \mathrm{sub}-$ jects who were identified from the company's medical records, personnel lists, and lists of urinary $\mathrm{Hg}$ measurements from the biological surveillance, which commenced in 1948.

Seven subjects were deceased and five had moved to distant parts of the country and were thus not considered for participation. One subject had died from epilepsy. Exclusion criteria included alcohol abuse, major head injuries, metabolic disorders, and neurological, psychiatric, or other diseases causing severe disability. Two subjects were excluded because of neurological diseases, one due to episodes of cerebral ischaemia and one due to encephalopathy and epilepsy diagnosed one year after the start of exposure to $\mathrm{Hg}$. He had also been heavily exposed to organic solvents. Six subjects were excluded due to alcohol abuse. Daily exposure to organic solvents for more than five years led to exclusion of nine subjects. Altogether, 24 exposed subjects were excluded from participation.

The reference group comprised men employed by the same company who worked in the same industrial complex as the exposed workers. They were randomly selected from employees in a nitrate fertiliser plant and frequency matched for age. They had experienced no known work related exposure to $\mathrm{Hg}$ compounds. The same criteria for exclusion were used for the referents as for the exposed subjects.

The response rate was $82 \cdot 5 \%(\mathrm{n}=77)$ among the previously exposed workers and $86.3 \%(n=$ 53) among the referents. The examinations were performed during 1989.

The mean age among the exposed subjects and referents was $44 \cdot 8$ (range $24 \cdot 2-64 \cdot 8$ ) and $45 \cdot 8$ (range 24.3-63.7) years respectively. The exposed subjects had an average of 9.6 years of education $v$ 9.5 years among the referents. The prevalence of current smoking was $57 \%$ and $68 \%$ respectively. The self reported mean alcohol consumption (converted to units of pure alcohol) was 0.3 (range $0-2.0$ ) $1 /$ month and 0.2 (range $0-0.9$ ) $1 /$ month among the exposed subjects and referents respectively. Among the exposed subjects, $58 \%$ had main- ly been employed as maintenance workers and $42 \%$ as production workers.

The groups under study were further characterised by current mean concentrations of $\mathrm{Hg}$ in whole blood $(\mathrm{B}-\mathrm{Hg})$ and urine $(\mathrm{U}-\mathrm{Hg})$ of 26.4 (median 25.0, range $12 \cdot 0-61 \cdot 0$ ) $\mathrm{nmol} / 1$ and $1 \cdot 8$ (median $1 \cdot 5$, range $0 \cdot 3-6 \cdot 1$ ) $\mathrm{nmol} / \mathrm{mmol}$ creatinine among the exposed subjects respectively, compared with 28.3 (median 26.0 , range $15 \cdot 0-64.0$ ) $\mathrm{nmol} / 1$ and 1.3 (median 1.2 , range $0.3-3.8$ ) $\mathrm{nmol} / \mathrm{mmol}$ creatinine among the referents. For comparison, the U-Hg not corrected for creatinine was on average $24.3 \mathrm{nmol} / 1$ among the previously exposed workers and $15 \cdot 2 \mathrm{nmol} / \mathrm{l}$ among the referents.

\section{INDIVIDUAL EXPOSURE ASSESSMENT}

Until the early 1970s, mercury was usually measured in two to four urine specimens each year for workers with the presumed highest exposure. Subsequently the frequency was reduced to two or three measurements each year. The compiled lists of these results were complete from 1952 to 1987 . Measurements covering the period from 1948 to 1952 were found in the individual medical records at the plant's health department.

Results from more than 2300 urinary $\mathrm{Hg}$ measurements were identified among the 77 exposed subjects. Based on these data, a cumulative "dose" of urinary $\mathrm{Hg}$ (cumulative $\mathrm{U}-\mathrm{Hg}$ ) was calculated for each worker. When no quarterly measurements were available, the mean of the two known measurements before and after the missing value was used. The urinary $\mathrm{Hg}$ concentration for each year was calculated as the mean of the four quarters. These concentrations were added for each year employed, giving a value for cumulative $\mathrm{U}-\mathrm{Hg}$.

Until 1975, different modifications of the dithizone method were used to determine the urinary $\mathrm{Hg}$ concentrations. Cold vapour atomic absorption spectrophotometry was introduced in 1975. Urinary creatinine concentrations were determined since 1979 to correct for urinary dilution. As the exposure among the participants had occurred mainly before 1979 , the cumulative $\mathrm{U}-\mathrm{Hg}$ is expressed in uncorrected terms.

The working atmosphere has been monitored since late 1953 by $\mathrm{Hg}$ vapour detectors, thereby determining the concentration of $\mathrm{Hg}$ in air $(\mathrm{A}-\mathrm{Hg})$ directly. The measurements were performed routinely at different sites in the cell room and the basement underneath the cell room. Quarterly mean A-Hgs were calculated by plant personnel and these figures were available to us. Based on these quarterly mean A-Hgs, a cumulative dose of $\mathrm{Hg}$ in air (cumulative $\mathrm{A}-\mathrm{Hg}$ ) was calculated as the sum of A-Hg for each employment year. The mean A-Hg calculated for 1954 was used as a substitute for the concentrations from 1947 to 1953. 
Table 1 Exposure related characteristics among 77 subjects previously exposed to $\mathrm{Hg}$ vapour

\begin{tabular}{|c|c|c|c|}
\hline & Mean & Median & Range \\
\hline $\begin{array}{l}\text { Current U-Hg (nmol/mmol } \\
\text { creatinine) }\end{array}$ & $1 \cdot 8$ & 1.5 & $0 \cdot 3-6 \cdot 1$ \\
\hline Current B-Hg (nmol/l) & $26 \cdot 4$ & $25 \cdot 0$ & $12 \cdot 0-61 \cdot 0$ \\
\hline Years since first exposure & 20.9 & $21 \cdot 0$ & $4 \cdot 0-41 \cdot 0$ \\
\hline Years since last exposure & $12 \cdot 3$ & $9 \cdot 0$ & $1 \cdot 0-35 \cdot 0$ \\
\hline Duration of exposure (y) & 70 & $5 \cdot 8$ & $1 \cdot 1-36 \cdot 2$ \\
\hline Cumulative U-Hg (nmol/1) & 3225 & 2287 & $167-17229$ \\
\hline $\begin{array}{l}\text { Cumulative U-Hg/year } \\
(\mathrm{nmol} / \mathrm{l})\end{array}$ & 531 & 402 & $41-2921$ \\
\hline $\begin{array}{l}\text { Cumulative A-Hg }\left(\mu \mathrm{g} / \mathrm{m}^{3}\right) \\
\text { Cumulative A-Hg/year }\left(\mu \mathrm{g} / \mathrm{m}^{3}\right)\end{array}$ & $\begin{array}{r}399 \\
59\end{array}$ & $\begin{array}{r}269 \\
49\end{array}$ & $\begin{array}{l}34-2260 \\
10-162\end{array}$ \\
\hline
\end{tabular}

Table 1 presents some exposure related indices among the exposed workers. They had been free from $\mathrm{Hg}$ vapour exposure for an average of 12.3 years (median 9.0). The individual mean $\mathrm{U}-\mathrm{Hg}$ for each year employed was $531 \mathrm{nmol} \mathrm{Hg} / 1$ (median 402), and the individual mean $\mathrm{A}-\mathrm{Hg}$ for each year employed was $59 \mu \mathrm{g} \mathrm{Hg} / \mathrm{m}^{3}$ (median 49). The mean duration of exposure was 7.9 years (median 5.8 ). Eighty six per cent of the workers had exceeded a urinary peak level of $500 \mathrm{nmol} / 1$ at least once during their time of exposure, whereas a value above $3000 \mathrm{nmol} / 1$ has been recorded for $16 \%$ of the subjects during their time of biological surveillance.

The exposed non-responders had been exposed to $\mathrm{Hg}$ vapour for an average of $8 \cdot 1$ (range $1 \cdot 3-34 \cdot 6$ ) years and their cumulative $\mathrm{U}-\mathrm{Hg}$ was 3022 (range 308-11 547) $\mathrm{nmol} \mathrm{Hg} / 1 v 3225 \mathrm{nmol} \mathrm{Hg} / \mathrm{l}$ in the group under study.

\section{EXAMINATIONS}

All subjects underwent a clinical interview with emphasis on work history, exposure to known occupational neurotoxicants, lifestyle habits, and medical history. A brief clinical examination was subsequently conducted. Specimens of urine were sampled to determine the current $\mathrm{U}-\mathrm{Hg}$. A blood sample was obtained for determination of the current B-Hg. Details of sampling and determination of $\mathrm{U}-\mathrm{Hg}$ and $\mathrm{B}-\mathrm{Hg}$ have been reported elsewhere. ${ }^{23}$

The neurological and neurophysiological examinations were accomplished by two trained neurologists. The examiners had neither knowledge of exposure state, nor amount of previous exposure. The neurography included a sensory study of the sural and median nerves and a motor study of the peroneal and median nerves. The evoked response examinations included pattern reversal visual evoked response, brainstem auditory evoked response, and somatosensory evoked response. A neurological state was established. Details describing the neurological and neurophysiological examinations will be reported elsewhere. ${ }^{22}$

\section{STATISTICS}

The distribution of many continuous variables was skewed. Hence, a non-parametric test (MannWhitney) was used for the comparison of intergroup differences.

Least square regression analysis was used to study the relation between exposure related variables and the neurophysiological measurements. The amplitudes of the measurements were log transformed to achieve homoscedasticity, which also normalised most of the distributions. The cumulative dose estimates were also log transformed as few exposed subjects had high cumulative doses, thus reducing the influence of these observations. To describe the relation between a dependent continuous variable and several independent variables, multiple stepwise (forward) linear regression analysis was performed ( $\mathrm{F}$ to enter $=$ $4 \cdot 0$, tolerance level $0 \cdot 01) .{ }^{24}$

Logistic regression analysis was used to study the relation between exposure related variables and

Table 2 Exposure related indices among 10 subjects previously exposed to $\mathrm{Hg}$ vapour with reduced distal sensation (A) and 15 exposed worker: with postural tremor/impaired coordination (B) compared with exposed subjects without such signs

\begin{tabular}{|c|c|c|c|c|c|c|c|c|c|c|c|c|c|c|}
\hline & \multicolumn{6}{|c|}{ Distal sensation } & \multirow[b]{3}{*}{ p Value } & \multicolumn{6}{|c|}{ Postural tremor/impaired coordination } & \multirow[b]{3}{*}{ p Valu } \\
\hline & \multicolumn{3}{|c|}{ Reduced $(n=10)$} & \multicolumn{3}{|c|}{ Not reduced $(n=67)$} & & \multicolumn{3}{|c|}{ Present $(n=15)$} & \multicolumn{3}{|c|}{ Not present $(n=62)$} & \\
\hline & Mean & Median & (Range) & Mean & Median & (Range) & & Mean & Median & (Range) & Mean & Median & (Range) & \\
\hline $\begin{array}{l}\text { Age (y) } \\
\text { U-Hg (nmol } \\
/ \text { /mmol) }\end{array}$ & $\begin{array}{l}48 \cdot 3 \\
2 \cdot 0\end{array}$ & $\begin{array}{l}49 \cdot 4 \\
1 \cdot 7\end{array}$ & $\begin{array}{l}(36 \cdot 3-63 \cdot 0) \\
(0 \cdot 5-6 \cdot 1)\end{array}$ & $\begin{array}{l}44 \cdot 3 \\
1 \cdot 8\end{array}$ & $\begin{array}{l}44 \cdot 1 \\
1 \cdot 4\end{array}$ & $\begin{array}{l}(24 \cdot 2-64 \cdot 8) \\
(0 \cdot 3-5 \cdot 2)\end{array}$ & $\begin{array}{l}0.29 \\
0.98\end{array}$ & $\begin{array}{l}50 \cdot 5 \\
1 \cdot 3\end{array}$ & $\begin{array}{l}55 \cdot 3 \\
1 \cdot 1\end{array}$ & $\begin{array}{l}(24 \cdot 4-64 \cdot 6) \\
(0 \cdot 4-2 \cdot 7)\end{array}$ & $\begin{array}{l}43 \cdot 5 \\
2 \cdot 0\end{array}$ & $\begin{array}{l}41 \cdot 0 \\
1.6\end{array}$ & $\begin{array}{l}(24 \cdot 2-64 \cdot 8) \\
(0 \cdot 3-6 \cdot 1)\end{array}$ & $\begin{array}{l}0.04 \\
0 \cdot 10\end{array}$ \\
\hline $\begin{array}{l}\text { B-Hg (nmol/) } \\
\text { Years exposed } \\
\text { Years since }\end{array}$ & $\begin{array}{l}26 \cdot 6 \\
13.9\end{array}$ & $\begin{array}{l}24 \cdot 5 \\
11 \cdot 5\end{array}$ & $\begin{array}{l}(20 \cdot 0-38 \cdot 0) \\
(2 \cdot 0-36 \cdot 2)\end{array}$ & $\begin{array}{l}26 \cdot 3 \\
7 \cdot 0\end{array}$ & $\begin{array}{l}25 \cdot 0 \\
5 \cdot 7\end{array}$ & $\begin{array}{l}(12 \cdot 0-61 \cdot 0) \\
(1 \cdot 1-25 \cdot 5)\end{array}$ & $\begin{array}{l}0.78 \\
0.03\end{array}$ & $\begin{array}{l}26 \cdot 3 \\
8 \cdot 8\end{array}$ & $\begin{array}{l}23 \cdot 0 \\
6 \cdot 2\end{array}$ & $\begin{array}{l}(18 \cdot 0-61 \cdot 0) \\
(1 \cdot 3-36 \cdot 2)\end{array}$ & $\begin{array}{l}26 \cdot 4 \\
7 \cdot 8\end{array}$ & $\begin{array}{l}26 \cdot 0 \\
5 \cdot 7\end{array}$ & $\begin{array}{l}(12 \cdot 0-44 \cdot 0) \\
(1 \cdot 1-25 \cdot 5)\end{array}$ & $\begin{array}{l}0.50 \\
0.75\end{array}$ \\
\hline $\begin{array}{l}\text { first exposure } \\
\text { last exposure } \\
\text { Cumulative }\end{array}$ & $\begin{array}{l}22 \cdot 7 \\
8 \cdot 4\end{array}$ & $\begin{array}{l}22 \cdot 0 \\
3 \cdot 5\end{array}$ & $\begin{array}{l}(11 \cdot 0-37 \cdot 0) \\
(1 \cdot 0-23 \cdot 0)\end{array}$ & $\begin{array}{l}20 \cdot 7 \\
12 \cdot 9\end{array}$ & $\begin{array}{l}21 \cdot 0 \\
11 \cdot 0\end{array}$ & $\begin{array}{l}(4 \cdot 0-41 \cdot 0) \\
(1 \cdot 0-35 \cdot 0)\end{array}$ & $\begin{array}{l}0.48 \\
0.29\end{array}$ & $\begin{array}{l}25 \cdot 6 \\
16.7\end{array}$ & $\begin{array}{l}28 \cdot 0 \\
23 \cdot 0\end{array}$ & $\begin{array}{l}(7 \cdot 0-37 \cdot 0) \\
(1 \cdot 0-33 \cdot 0)\end{array}$ & $\begin{array}{l}19 \cdot 8 \\
11 \cdot 3\end{array}$ & $\begin{array}{l}20 \cdot 0 \\
7 \cdot 0\end{array}$ & $\begin{array}{l}(4 \cdot 0-41 \cdot 0) \\
(1 \cdot 0-35 \cdot 0)\end{array}$ & $\begin{array}{l}0.04 \\
0.08\end{array}$ \\
\hline $\begin{array}{l}\mathrm{U}-\mathrm{Hg}(\mathrm{nmol} / \mathrm{l}) \\
\mathrm{A}-\mathrm{Hg}\left(\mu \mathrm{g} / \mathrm{m}^{3}\right)\end{array}$ & $\begin{array}{l}5621 \\
692\end{array}$ & $\begin{array}{l}3186 \\
484\end{array}$ & $\begin{array}{l}(918-17229) \\
(197-2260)\end{array}$ & $\begin{array}{l}2868 \\
355\end{array}$ & $\begin{array}{l}2185 \\
268\end{array}$ & $\begin{array}{l}(167-14309) \\
(34-1555)\end{array}$ & $\begin{array}{l}0.06 \\
0.04\end{array}$ & $\begin{array}{l}3932 \\
450\end{array}$ & $\begin{array}{l}2209 \\
268\end{array}$ & $\begin{array}{l}(517-17229) \\
(67-2260)\end{array}$ & $\begin{array}{l}3054 \\
386\end{array}$ & $\begin{array}{l}2375 \\
298\end{array}$ & $\begin{array}{l}(167-14309) \\
(34-1555)\end{array}$ & $\begin{array}{r}0.82 \\
0.90\end{array}$ \\
\hline
\end{tabular}


dichotomous outcome variables. Due to small numbers, the maximum number of covariates included in any model was two. Both models with the continuous covariates included linearity and as sets of design variables representing different categories of covariable values were fitted. The model evaluations were based on likelihood ratio tests.

The level of significance was chosen at $5 \%$, and all $p$ values reported are two tailed. The statistical calculations were performed using the statistical data package BMDP PC 90 on a personal computer.

\section{Results}

Group comparisons between the exposed subjects and referents are reported separately. ${ }^{22}$ Among the 77 exposed subjects, 15 had impaired coordination and/or postural tremor, whereas 10 had slightly reduced distal sensation. Only one subject had both postural tremor/impaired coordination and reduced distal sensation, and one subject had impaired coordination without postural tremor. Table 2 shows some exposure related indices among the exposed subjects with neurological abnormalities compared with exposed subjects without such signs. Cumulative $\mathrm{A}-\mathrm{Hg}$ was higher and the duration of exposure longer among the exposed subjects with reduced distal sensation compared with subjects without this sign. The subjects with tremor or impaired coordination were older and were exposed for the first time earlier than subjects without these findings. Current $\mathrm{B}-\mathrm{Hg}$ and $\mathrm{U}-\mathrm{Hg}$ did not differ between the groups.

The neurophysiological results among exposed subjects with neurological abnormalities were com- pared with the results for the referents, and table 3 presents the results from the evoked response examinations for the exposed subjects with postural tremor/impaired coordination. They had bilateral prolongation of $\mathrm{P} 100$ and unilateral prolongation of N75 and N145 on the right side. Unilaterally shorter latencies were found for N9-N13 and N13-N20 in the somatosensory evoked response. None of the neurographical measurements differed significantly between these exposed subjects and the referents. No significant differences were found for brainstem auditory evoked response.

Table 4 presents the results from the neurographical measurements among the exposed subjects with reduced distal sensation compared with the referents. Reduced median sensory nerve conduction velocity and significantly shorter unilateral N13-N20 latency compared with the referents (mean 5.7, range $4 \cdot 7-6 \cdot 4$, not tabulated) were found. None of the other results from the evoked response examinations differed significantly between the exposed subjects with reduced distal sensation and the referents.

Table 5 shows the univariate relations expressed as Pearson's correlation coefficients between selected neurophysiological results and age, current B$\mathrm{Hg}$, current $\mathrm{U}-\mathrm{Hg}$, cumulative $\mathrm{U}-\mathrm{Hg}$, cumulative $\mathrm{A}-\mathrm{Hg}$, peak levels (number of times recorded when urinary mercury exceeded $1000 \mathrm{nmol} / \mathrm{l}$ ), and years since first exposure to $\mathrm{Hg}$. No significant associations between visual evoked response and age, current $\mathrm{B}-\mathrm{Hg}$, or current $\mathrm{U}-\mathrm{Hg}$ were seen among the referents. In this group the conduction velocities of the median and peroneal motor nerve and the median sensory nerve were correlated with age, as were the amplitudes of the sensory median and

Table 3 Selected results from the visual evoked response and somatosensory evoked response among referents, exposed subjects with tremor/impaired coordination, and all exposed subjects

\begin{tabular}{|c|c|c|c|c|c|c|c|c|c|}
\hline & \multicolumn{3}{|c|}{ Referents $(n=53)$} & \multicolumn{3}{|c|}{$\begin{array}{l}\text { Tremorlimpaired } \\
\text { coordination }(n=15)\end{array}$} & \multicolumn{3}{|c|}{ All exposed $(n=77)$} \\
\hline & Mean & $S D$ & Median & Mean & $S D$ & Median & Mean & $S D$ & Median \\
\hline \multicolumn{10}{|c|}{ Visual evoked response } \\
\hline $\begin{array}{l}\text { N75 r } \\
\text { N75 } 1 \\
\text { P100 r } \\
\text { P100 } 1 \\
\text { N145 r } \\
\text { N145 } 1 \\
\text { A1 r } \\
\text { A1 } 1 \\
\text { A2 r } \\
\text { A2 } 1\end{array}$ & $\begin{array}{r}77 \cdot 4 \\
77 \cdot 0 \\
103 \cdot 1 \\
103 \cdot 7 \\
136 \cdot 7 \\
138 \cdot 2 \\
8 \cdot 3 \\
8 \cdot 0 \\
8 \cdot 6 \\
9 \cdot 2\end{array}$ & $\begin{array}{r}4 \cdot 4 \\
4 \cdot 0 \\
4 \cdot 8 \\
4 \cdot 4 \\
13 \cdot 4 \\
12 \cdot 8 \\
4 \cdot 0 \\
3 \cdot 9 \\
4 \cdot 1 \\
4 \cdot 8\end{array}$ & $\begin{array}{r}76 \cdot 8 \\
76 \cdot 2 \\
103 \cdot 0 \\
104.0 \\
135 \cdot 0 \\
135 \cdot 0 \\
7 \cdot 7 \\
6 \cdot 8 \\
7 \cdot 8 \\
8 \cdot 0\end{array}$ & $\begin{array}{c}80 \cdot 1^{\star} \\
78 \cdot 9 \\
107 \cdot 4^{\star} \\
107 \cdot 6^{\star} \\
141 \cdot 3^{\star} \\
141 \cdot 9 \\
8 \cdot 7 \\
8 \cdot 4 \\
9 \cdot 5 \\
9.9\end{array}$ & $\begin{array}{l}3 \cdot 6 \\
5 \cdot 4 \\
5 \cdot 5 \\
5 \cdot 3 \\
8 \cdot 2 \\
8 \cdot 6 \\
5 \cdot 1 \\
5 \cdot 0 \\
5 \cdot 3 \\
5 \cdot 9\end{array}$ & $\begin{array}{r}79 \cdot 1 \\
76 \cdot 8 \\
106 \cdot 0 \\
108 \cdot 0 \\
140.0 \\
144.0 \\
7 \cdot 3 \\
6.4 \\
9.2 \\
9.0\end{array}$ & $\begin{array}{r}78 \cdot 3 \\
78 \cdot 4 \\
104 \cdot 2 \\
104 \cdot 5 \\
137 \cdot 8 \\
139 \cdot 3 \\
8 \cdot 3 \\
7 \cdot 9 \\
9 \cdot 1 \\
9 \cdot 1\end{array}$ & $\begin{array}{r}4 \cdot 0 \\
4 \cdot 4 \\
5 \cdot 1 \\
5 \cdot 0 \\
14 \cdot 0 \\
13 \cdot 1 \\
3 \cdot 9 \\
3 \cdot 8 \\
4 \cdot 1 \\
4 \cdot 4\end{array}$ & $\begin{array}{r}77 \cdot 3 \\
77 \cdot 3 \\
103 \cdot 0 \\
104 \cdot 0 \\
134 \cdot 0 \\
137 \cdot 0 \\
7 \cdot 5 \\
7 \cdot 0 \\
8 \cdot 7 \\
8 \cdot 5\end{array}$ \\
\hline \multicolumn{10}{|c|}{ Somatosensory evoked response } \\
\hline $\begin{array}{l}\text { N9N13 r } \\
\text { N9N131 } \\
\text { N13N20 r }\end{array}$ & $\begin{array}{l}4 \cdot 0 \\
4 \cdot 0 \\
6 \cdot 2\end{array}$ & $\begin{array}{l}0.5 \\
0.5 \\
0.6\end{array}$ & $\begin{array}{l}4 \cdot 0 \\
4 \cdot 0 \\
6 \cdot 1\end{array}$ & $\begin{array}{l}3 \cdot 7^{\star} \\
3.9 \\
5 \cdot 9 \\
5.9 \star\end{array}$ & $\begin{array}{l}0.5 \\
0.4 \\
0.7\end{array}$ & $\begin{array}{l}3.8 \\
3.9 \\
5.9\end{array}$ & $\begin{array}{l}3.8 \\
3.9 \\
6.1\end{array}$ & $\begin{array}{l}0.5 \\
0.5 \\
0.7\end{array}$ & $\begin{array}{l}3.8 \\
3.8 \\
6.0\end{array}$ \\
\hline
\end{tabular}

${ }^{\star} \mathrm{p}<0.05$ (between the referents and the two exposed groups). All latencies measured in ms and all amplitudes (A) in $\mu \mathrm{V}$. 
Table 4 Selected neurographical results among referents, exposed subjects with reduced distal sensation, and all exposed subjects

\begin{tabular}{|c|c|c|c|c|c|c|c|c|c|}
\hline & \multicolumn{3}{|c|}{$\begin{array}{l}\text { Referents } \\
(n=53)\end{array}$} & \multicolumn{3}{|c|}{$\begin{array}{l}\text { Reduced sensation } \\
(n=10)\end{array}$} & \multicolumn{3}{|c|}{$\begin{array}{l}\text { All exposed } \\
(n=77)\end{array}$} \\
\hline & Mean & $S D$ & Median & Mean & $S D$ & Median & Mean & $S D$ & Median \\
\hline $\begin{array}{l}\text { Sensory median nerve: } \\
\text { NCV }(\mathrm{m} / \mathrm{s}) \\
\text { A }(\mu V) \\
\text { Sensory sural nerve: }\end{array}$ & $\begin{array}{l}59 \cdot 0 \\
10 \cdot 5\end{array}$ & $\begin{array}{l}5 \cdot 2 \\
5 \cdot 5\end{array}$ & $\begin{array}{r}59 \cdot 8 \\
9 \cdot 1\end{array}$ & $\begin{array}{c}54 \cdot 6^{\star} \\
9 \cdot 8\end{array}$ & $\begin{array}{l}6 \cdot 5 \\
5 \cdot 3\end{array}$ & $\begin{array}{r}53 \cdot 7 \\
8 \cdot 7\end{array}$ & $\begin{array}{l}57 \cdot 4 \\
10 \cdot 3\end{array}$ & $\begin{array}{l}6 \cdot 2 \\
5 \cdot 7\end{array}$ & $\begin{array}{r}57 \cdot 9 \\
9 \cdot 1\end{array}$ \\
\hline $\begin{array}{l}\text { NCV }(\mathrm{m} / \mathrm{s}) \\
\text { A }(\mu \mathrm{V}) \\
\text { Motor median nerve: }\end{array}$ & $\begin{array}{r}49 \cdot 0 \\
5 \cdot 1\end{array}$ & $\begin{array}{l}5 \cdot 3 \\
3 \cdot 8\end{array}$ & $\begin{array}{r}49 \cdot 1 \\
4 \cdot 5\end{array}$ & $\begin{array}{r}49 \cdot 6 \\
3.9\end{array}$ & $\begin{array}{l}5 \cdot 2 \\
4 \cdot 5\end{array}$ & $\begin{array}{r}49 \cdot 0 \\
2 \cdot 5\end{array}$ & $\begin{array}{r}50 \cdot 2 \\
7 \cdot 3\end{array}$ & $\begin{array}{l}4 \cdot 5 \\
5 \cdot 7\end{array}$ & $\begin{array}{r}50 \cdot 2 \\
5 \cdot 6\end{array}$ \\
\hline $\begin{array}{l}\mathrm{NCV}(\mathrm{m} / \mathrm{s}) \\
\mathrm{A}(\mathrm{mV}) \\
\text { Motor peroneal nerve: }\end{array}$ & $\begin{array}{r}58 \cdot 0 \\
6 \cdot 8\end{array}$ & $\begin{array}{l}4 \cdot 8 \\
2 \cdot 5\end{array}$ & $\begin{array}{r}58 \cdot 7 \\
7 \cdot 0\end{array}$ & $\begin{array}{r}59 \cdot 3 \\
5 \cdot 8\end{array}$ & $\begin{array}{l}5 \cdot 8 \\
2 \cdot 9\end{array}$ & $\begin{array}{c}60 \cdot 0 \\
5 \cdot 9\end{array}$ & $\begin{array}{r}58 \cdot 8 \\
6 \cdot 1\end{array}$ & $\begin{array}{l}5 \cdot 1 \\
2 \cdot 6\end{array}$ & $\begin{array}{r}58 \cdot 7 \\
5 \cdot 8\end{array}$ \\
\hline $\begin{array}{l}\mathrm{NCV}(\mathrm{m} / \mathrm{s}) \\
\mathrm{A}(\mathrm{mV})\end{array}$ & $\begin{array}{r}45 \cdot 9 \\
3 \cdot 6\end{array}$ & $\begin{array}{l}5 \cdot 0 \\
2 \cdot 1\end{array}$ & $\begin{array}{r}46 \cdot 0 \\
3 \cdot 3\end{array}$ & $\begin{array}{r}45 \cdot 4 \\
4 \cdot 3\end{array}$ & $\begin{array}{l}4 \cdot 3 \\
2 \cdot 6\end{array}$ & $\begin{array}{r}46 \cdot 7 \\
4 \cdot 4\end{array}$ & $\begin{array}{r}47 \cdot 0 \\
3 \cdot 5\end{array}$ & $\begin{array}{l}4 \cdot 7 \\
2 \cdot 5\end{array}$ & $\begin{array}{r}47 \cdot 3 \\
2 \cdot 9\end{array}$ \\
\hline
\end{tabular}

${ }^{\star} \mathrm{p}<0.05$ (between referents and the two exposed groups). NCV $=$ Nerve conduction velocity, $A=$ amplitude.

Table 5 Univariate correlation coefficients (Pearson) between selected neurophysiological and independent variables among 77 exposed workers and 53 referents

\begin{tabular}{|c|c|c|c|c|}
\hline \multirow[b]{2}{*}{$\begin{array}{l}\text { Exposed: } \\
\text { Age } \\
\text { Current U-Hg } \\
\text { Current B-Hg } \\
\text { Cumulative A-Hg } \\
\text { Cumulative U-Hg } \\
\text { Peak values } \\
\text { Years since } \\
\quad \text { first exposure }\end{array}$} & \multicolumn{2}{|c|}{ Visual evoked response } & \multicolumn{2}{|c|}{ Pattern reversal } \\
\hline & $\begin{array}{l}\text { N75 r } \\
0.43^{\star \star \star} \\
-0.33^{\star \star} \\
-0 \cdot 14 \\
0 \cdot 09 \\
0 \cdot 20 \\
0 \cdot 26^{\star} \\
0.44^{\star \star \star}\end{array}$ & 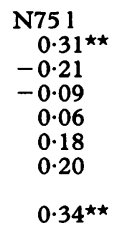 & $\begin{array}{c}\log A 1 \mathbf{r} \\
-0.23^{\star} \\
0.18 \\
0.20 \\
-0.15 \\
-0.06 \\
-0.10\end{array}$ & $\begin{array}{l}\log A 11 \\
-0.25^{\star} \\
0.16 \\
0.27^{\star} \\
-0.19 \\
-0.09 \\
-0.05 \\
-0.36^{\star \star}\end{array}$ \\
\hline $\begin{array}{l}\text { Referents: } \\
\text { Age } \\
\text { Current U-Hg } \\
\text { Current B-Hg }\end{array}$ & $\begin{array}{l}0.19 \\
0.04 \\
0.11\end{array}$ & $\begin{array}{r}0.16 \\
-0.04 \\
0.04\end{array}$ & $\begin{array}{l}-0.12 \\
-0.11 \\
-0.14\end{array}$ & $\begin{array}{l}-0.07 \\
-0.03 \\
-0.18\end{array}$ \\
\hline Exposed: & $\begin{array}{l}\text { ographical mo } \\
\text { Median ner }\end{array}$ & $\begin{array}{l}\text { otor nerve mec } \\
\text { rve }\end{array}$ & zsurements & en \\
\hline $\begin{array}{l}\text { Age } \\
\text { Current U-Hg } \\
\text { Current B-Hg } \\
\text { Cumulative A-Hg } \\
\text { Cumulative U-Hg } \\
\text { Peak values } \\
\text { Years since }\end{array}$ & $\begin{array}{l}-0.42^{\star \star \star} \\
0.22 \\
-0.01 \\
-0 \cdot 22 \\
-0.35^{\star \star} \\
-0 \cdot 20\end{array}$ & $\begin{array}{c}\log A \\
0.13 \\
0.03 \\
0.01 \\
-0.02 \\
0.09 \\
0.22\end{array}$ & $\begin{array}{l}\text { NCV } \\
-0.28 \star \\
0.10 \\
0.08 \\
-0.21 \\
-0.19 \\
-0.11\end{array}$ & $\begin{array}{l}-0.31^{\star \star} \\
0.32^{\star \star} \\
-0.04 \\
-0.02 \\
-0.04 \\
0.07\end{array}$ \\
\hline $\begin{array}{l}\text { Referents: } \\
\text { Age } \\
\text { Current U-Hg } \\
\text { Current B-Hg }\end{array}$ & $\begin{array}{l}-0.42^{\star \star} \\
0.02 \\
-0.24\end{array}$ & $\begin{array}{r}-0 \cdot 16 \\
0 \cdot 10 \\
-0 \cdot 15\end{array}$ & $\begin{array}{l}-0.43^{\star \star} \\
-0.15 \\
-0.20\end{array}$ & $\begin{array}{l}-0.42^{\star \star} \\
-0.07 \\
-0.06\end{array}$ \\
\hline \multicolumn{5}{|c|}{ Neurographical sensory nerve measurements } \\
\hline $\begin{array}{l}\text { Age } \\
\text { Current U-Hg } \\
\text { Current B-Hg } \\
\text { Cumulative A-Hg } \\
\text { Cumulative U-Hg } \\
\text { Peak values } \\
\text { Years since }\end{array}$ & $\begin{array}{l}\text { NCV } \\
-0.32^{\star \star} \\
0.39^{\star \star \star} \\
0.02 \\
-0.23^{\star} \\
-0.14 \\
-0.05\end{array}$ & $\begin{array}{l}\log \mathrm{A} \\
-0 \cdot 53^{\star \star \star} \\
0 \cdot 41^{\star \star \star} \\
0 \cdot 10 \\
-0 \cdot 16 \\
-0 \cdot 20 \\
-0 \cdot 18\end{array}$ & $\begin{array}{l}\text { NCV } \\
-0.05 \\
-0.08 \\
-0.22 \\
-0.06 \\
-0.11 \\
-0.05\end{array}$ & $\begin{array}{l}\log A \\
-0.33^{\star \star} \\
0 \cdot 13 \\
0 \cdot 14 \\
-0.29^{\star} \\
-0 \cdot 36^{\star \star} \\
-0.33^{\star \star}\end{array}$ \\
\hline $\begin{array}{l}\text { first exposure } \\
\text { Referents: }\end{array}$ & $-0 \cdot 33^{\star \star}$ & -0.5 & 0.05 & $-0.35^{\star \star}$ \\
\hline $\begin{array}{l}\text { Age } \\
\text { Current U-Hg } \\
\text { Current B-Hg }\end{array}$ & $\begin{array}{l}-0.32^{\star} \\
-0.02 \\
-0.16\end{array}$ & $\begin{array}{l}-0.51^{\star \star \star} \\
0.13 \\
-0.17\end{array}$ & $\begin{array}{r}-0.11 \\
0.13 \\
-0.09\end{array}$ & $\begin{array}{c}-0.54^{\star \star \star} \\
0.08 \\
-0.17\end{array}$ \\
\hline
\end{tabular}

${ }^{\star} \mathrm{p}<0.05 ;{ }^{\star \star} \mathrm{p}<0.01 ;{ }^{\star \star \star} \mathrm{p}<0.001 ; \log \mathrm{A}=\log$ amplitude. sural nerve and motor peroneal nerve. There were no statistically significant associations between current $\mathrm{U}-\mathrm{Hg}$ or current $\mathrm{B}-\mathrm{Hg}$ and the neurophysiological measurements among the referents. Several variables in the visual evoked response were associated with age and years since first exposure among the exposed subjects. An association between age and several neurographical measures was also found. The amplitude and conduction velocity of the sensory median nerve and the amplitude of the peroneal nerve were positively correlated with the current $\mathrm{U}-\mathrm{Hg}$, indicating an increased amplitude or faster nerve conduction velocity with higher $\mathrm{U}-\mathrm{Hg}$. Some of the variables were also correlated with cumulative dose estimates.

Table 6 presents the results from the stepwise multiple linear regression analysis for the different effect indices. One model (model IV) was used for the referents and three alternative models for the exposed subjects. The table shows the variables that were included and the multiple $\mathbf{R}$ for the different models. All multiple Rs presented and the variables included were statistically significant. No models for the visual evoked response were established among the referents. Among the exposed subjects the models with the highest multiple $\mathbf{R}$ included years since first exposure, and the figure presents the relation between the latency of the first wave (N75) on the right side in the visual evoked response and the number of years since first exposure to $\mathrm{Hg}$. Age was the only significant independent variable included in the model for the neurography among the referents. The motor nerve measurements among the exposed subjects were mainly related to age, except for the peroneal amplitude, when U-Hg gave the highest multiple $\mathrm{R}$. Adding age to the model increased the multiple $\mathbf{R}$ to 0.38 with the contribution of age of borderline significance $(p=0.05)$. Sensory median nerve conduction velocity was related to current $\mathrm{U}-\mathrm{Hg}$ and cumulative $\mathrm{A}-\mathrm{Hg}$ (multiple $\mathrm{R}=0.51$ ) and 
Table 6 Multiple Rs from the stepwise multiple linear regression analysis among 77 subjects exposed to $\mathrm{Hg}$ vapour and 53 referents. Independent variables contributing statistical significance to the model in parentheses

\begin{tabular}{lllll}
\hline & Model I & Model II & Model III & \multicolumn{1}{c}{ Model IV } \\
& $R$ & $R$ & $R$ \\
\hline Visual evoked response: & $0.43(1)$ & $0.44(6)$ & $0.43(1)$ & - \\
N75 r & $0.31(1)$ & $0.34(6)$ & $0.31(1)$ & - \\
N75 1 & $0.23(1)$ & $0.30(6)$ & $0.23(1)$ & - \\
Log A1 r & $0.38(1,3)$ & $0.43(6,3)$ & $0.38(1,3)$ & - \\
Log A1 1 & 0.38 & \\
Neurographical motor nerve & measurements: & & \\
Median NCV & $0.42(1)$ & $0.36(6)$ & $0.42(1)$ & $0.42(1)$ \\
Median A & - & - & - & - \\
Peroneal NCV & $0.28(1)$ & $0.25(6)$ & $0.28(1)$ & $0.43(1)$ \\
Peroneal A & $0.32(2)$ & $0.32(2)$ & - & $0.42(1)$ \\
Neurographical sensory nerve measurements: & \\
Median NCV & $0.39(2)$ & $0.39(2)$ & $0.51(2,7)$ & $0.32(1)$ \\
Median A & $0.58(1,2)$ & $0.57(2,6)$ & $0.58(1,2)$ & $0.51(1)$ \\
Sural NCV & - & - & - & - \\
Sural A & $0.36(4)$ & $0.36(4)$ & $0.33(5)$ & $0.54(1)$ \\
\hline
\end{tabular}

All multiple Rs are statistically significant $(p<0.05)$;

$A=$ amplitude.

Independent variables used: 1 = age, 2 = current $\mathrm{U}-\mathrm{Hg}, 3=$ current $\mathrm{B}-\mathrm{Hg}, 4=$ cumulative $\mathrm{U}-\mathrm{Hg}, 5=$ urinary peak values > $1000 \mathrm{nmol} / \mathrm{l}, 6$ = years since first exposure, 7 = cumulative $\mathrm{A}-\mathrm{Hg}$.

Model I: Exposed subjects; variables tested; 1, 2, 3, 4, 5 .

Model II: Exposed subjects; variables tested; 1, 2, 3, 5, 6 .

Model III: Exposed subjects; variables tested; 1, 2, 3, 5, 7 .

Model IV: Referents; variables tested; 1, 2, 3.

sural amplitude to cumulative $\mathrm{U}-\mathrm{Hg}$.

The models with the highest multiple $R$ were used to study the influence of current alcohol consumption. Alcohol consumption did not contribute to any of the neurophysiological results among the referents. Among the exposed subjects the alcohol consumption contributed significantly $(p=0.02)$

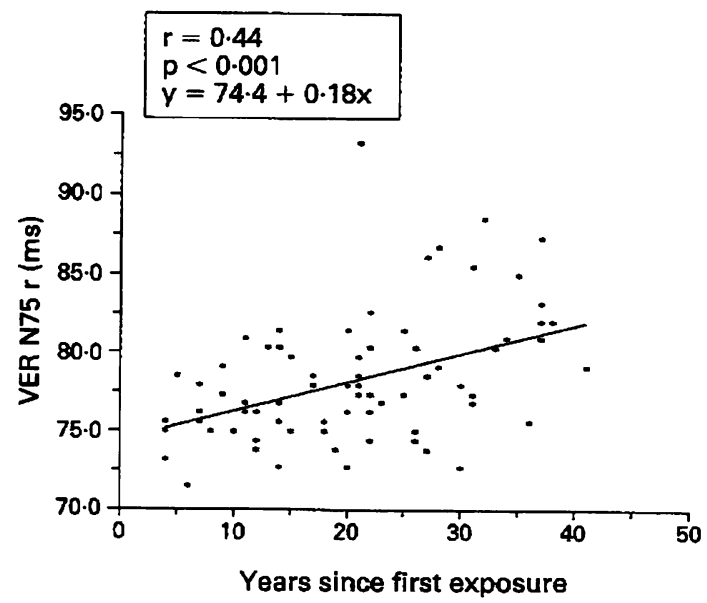

Relation between years since the start of exposure to $\mathrm{Hg}$ vapour and the results from N75, right side, in the visual evoked response (VER). to the right $\mathrm{Al}$ in the visual evoked response giving a multiple $R$ of 0.40 . The multiple $R$ was 0.47 on the left side $(p=0.08$, two tailed for alcohol consumption). The partial regression coefficients were positive. The median motor nerve conduction velocity was also related to the alcohol consumption with increasing consumption resulting in decreasing nerve conduction velocity.

Table 7 presents the final multiple linear regression equations for some of the neurophysiological variables studied among the previously exposed subjects. The addition of current consumption of smoking tobacco to these final models did not increase the multiple $R$. The brainstem auditory evoked response measurements were not related to any of the exposure indices.

Logistic regression analysis was performed on the neurological data to study the occurrence of postural tremor/impaired coordination and reduced distal sensation. The outcome variables were categorised as normal (0) or abnormal (1). Continuous independent variables were used. Table 8 presents the results of the univariate relations between the exposure related variables or age and the outcome of effect. Cumulative $\mathrm{U}-\mathrm{Hg}$ and cumulative $\mathrm{A}-\mathrm{Hg}$ were significantly associated with reduced distal sensation, whereas age and years since first exposure were not. Age and years since first exposure, but not the cumulative $\mathrm{U}-\mathrm{Hg}$ or cumulative $\mathrm{A}-\mathrm{Hg}$, were associated with postural tremor/impaired coordination. Models with more independent variables are not presented due to the few cases.

\section{Discussion}

Current $\mathrm{U}-\mathrm{Hg}$ and $\mathrm{B}-\mathrm{Hg}$ were low among the exposed subjects and comparable with the concentrations in the reference group, indicating that there was no current work related exposure to $\mathrm{Hg}$ among the subjects in the index group.

As indicated elsewhere, ${ }^{22}$ the prevalence of postural tremor, impaired coordination, and reduced distal sensation were increased among the exposed subjects compared with the referents. The present results indicate that the exposed subjects with postural tremor or impaired coordination were older and had been exposed to $\mathrm{Hg}$ vapour for the first time earlier than the other exposed subjects, whereas the exposed subjects with reduced distal sensation had higher cumulative $\mathrm{U}-\mathrm{Hg}$ and cumulative A-Hg than those without such abnormalities. These findings seem to be supported by the logistic regression analysis, where an association between the dose estimates (cumulative $\mathrm{U}-\mathrm{Hg}$, cumulative $\mathrm{A}-\mathrm{Hg}$ ) and reduced distal sensation but not postural tremor/impaired coordination, was found. Among 14 subjects with postural tremor, seven had impaired coordination also, whereas only one subject had reduced distal sensation, indicating that 
Table 7 The final multiple linear regression equations among 77 subjects previously exposed to Hg. $p$ Values for the independent variables included in brackets

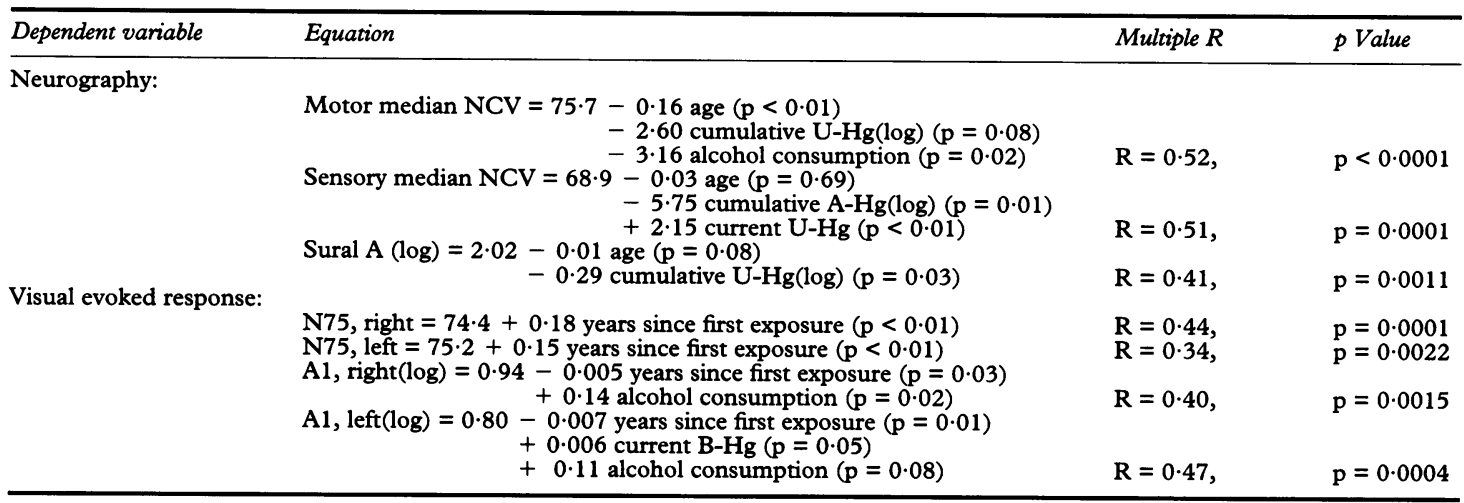

NCV = Nerve conduction velocity; $A=$ amplitude.

central nervous system signs in the present study were not associated with reduced distal sensation.

Exposed subjects with postural tremor/impaired coordination had prolongation of P100 bilaterally, and N75 and N145 unilaterally in the visual evoked response compared with the referents, which could indicate an involvement of both the visual pathway and cerebellum after previous exposure to $\mathrm{Hg}$ vapour. A study among 41 chloralkali workers under ongoing exposure to $\mathrm{Hg}$ vapour with computerised electroencephalography showed slower electroencephalographic complexes compared with the unexposed referents, most pronounced in the occipital region. ${ }^{10}$ Prolongation of P100 in the visual evoked response was seen in a study on 28 work- ers with suspected chronic $\mathrm{Hg}$ intoxication. ${ }^{8}$ The exact current urinary $\mathrm{Hg}$ concentrations among the exposed subjects in that study were not stated. The postmortem observations made by Takahata et al ${ }^{19}$ on two mercury miners more than 10 years after they ceased exposure showed the highest $\mathrm{Hg}$ concentrations in the occipital cortex and small granules of $\mathrm{Hg}$ in the nerve cells, particularly in the Purkinje cells.

In the present study the median sensory nerve conduction velocity was reduced among the exposed subjects with reduced distal sensation compared with the referents. Albers et $a l^{t}$ found reduced median sensory nerve conduction velocity among 18 chloralkali workers with mild poly-

Table 8 Results from logistic regression analysis among 77 previously exposed workers (univariate relations between neurological findings and independent variables)

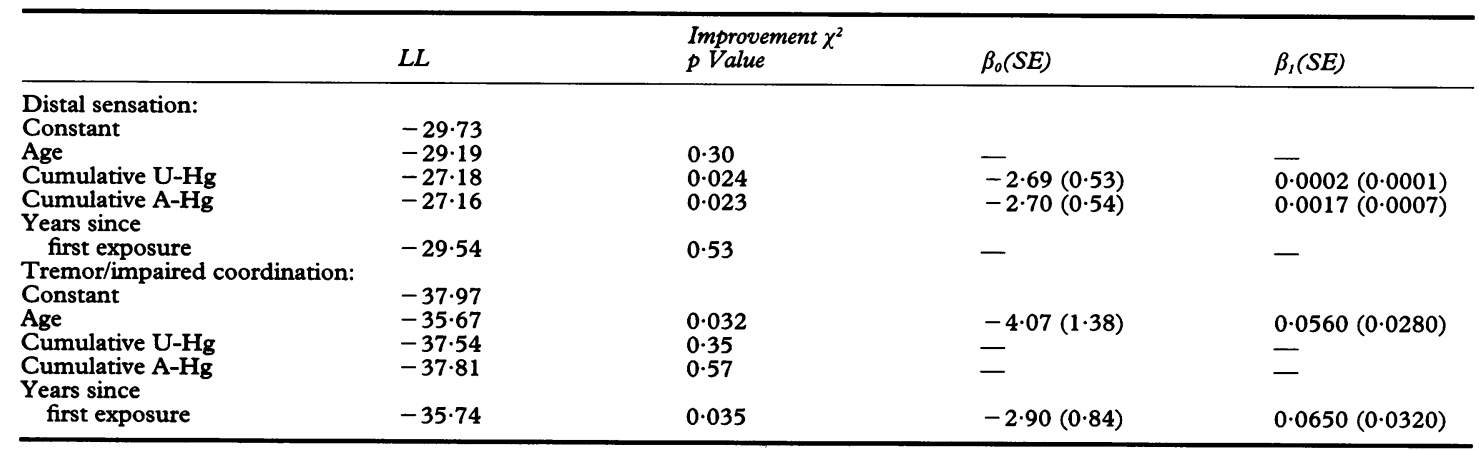

$\mathrm{LL}=$ Log-likelihood; neurological outcome variables were categorised as normal (0) or abnormal (1) based on the clinical neurological examination. 
neuropathy during ongoing exposure compared with 120 chloralkali workers without such findings.

In the examinations of somatosensory evoked response, a shorter central conduction time (N13N20) was found both among exposed subjects with postural tremor or impaired coordination and reduced distal sensation when compared with the referents. The reduction was statistically significant, however, only unilaterally. Shorter central conduction time has also been observed in another study among 11 chloralkali operators during ongoing exposure to $\mathrm{Hg},{ }^{12}$ and the authors suggested that damage in inhibiting mechanisms in the central sensory pathways could explain the results.

In the visual evoked response examinations in the present study, N75 and the amplitudes (A1) were bilaterally correlated with age among the exposed, but not among the referents. Slightly higher correlation coefficients were calculated when years since first exposure was used as an independent variable in the model. These findings may indicate that years since first exposure is a more appropriate predictor for these changes in visual evoked response than is age. It is difficult however, to differentiate between the two because of the high correlation between them.

A significant association between cumulative A$\mathrm{Hg}$ and median sensory nerve conduction velocity was found, whereas the association between cumulative $\mathrm{U}-\mathrm{Hg}$ and the median motor nerve conduction velocity was of borderline significance $(p=$ $0 \cdot 08$, two tailed). Shapiro et $a^{16}$ noted reduced median motor nerve conduction velocity among dentists during ongoing exposure to $\mathrm{Hg}$ vapour, with a high concentration of $\mathrm{Hg}$ at the wrist as determined by an $x$ ray fluorescence technique. The present results also indicated an association between cumulative $\mathrm{U}-\mathrm{Hg}$ and the amplitude of the sural nerve; however, other statistical procedures did not show any group differences for this amplitude.

Current alchohol consumption was significantly correlated with the median motor nerve conduction velocity in the exposed group, giving lower nerve conduction velocity with increasing present consumption. This effect was not found among the referents. A possible explanation could be that a nerve already subclinically influenced by past exposure to inorganic $\mathrm{Hg}$ is more vulnerable to other neurotoxins. Current alcohol consumption was also positively correlated with the amplitude (A1) of the visual evoked response in the multiple linear regression analysis. Assuming that the consumption at the time of the study was about the same as in the period when the subjects were exposed, a possible interpretation could be that alcohol consumption has led to reduced $\mathrm{Hg}$ retention in the brain. Hursh et $a l^{25}$ found a decreased concentration of mercury in the brain of rats given ethanol before exposure to $\mathrm{Hg}$ vapour compared with rats not given ethanol. In a study by Nylander et al ${ }^{26}$ on the relation between dental amalgam fillings and $\mathrm{Hg}$ retention in the brain, a somewhat lower than expected concentration of $\mathrm{Hg}$ in the occipital lobe was found in nine cases with suspected alcoholism based on the regression line between the number of tooth surfaces containing amalgam and the $\mathrm{Hg}$ concentration in the occipital lobe.

Minor effects associated with past exposure to $\mathrm{Hg}$ vapour on both the peripheral and central nervous system were found in the present study, which could indicate that neurological abnormalities may persist many years after exposure had ceased. The effects are modest, however, in clinical terms, as reported elsewhere. ${ }^{22}$ Persistent neurological abnormalities after ceasing exposure to $\mathrm{Hg}$ vapour was also noted by Albers et al ${ }^{17}$ who examined 247 workers exposed to elemental $\mathrm{Hg} 20$ to 35 years after exposure stopped. They found disturbed coordination, increased prevalence of tremor, and reduced sensation among the highest exposed subjects, observations in agreement with those of the present study. Ehrenberg et al ${ }^{15}$ studied 84 exposed workers manufacturing thermometers. They found more prevalent tremor, dysdiadochokinesia, abnormal Romberg's test and difficulty with heel to toe gait. Their findings were not related to recent exposure to $\mathrm{Hg}$, but there was some suggestion of an association with previous exposure.

The observation in the present study of postural tremor or impaired coordination, together with abnormalities in some aspects of the visual evoked response among the exposed subjects, could indicate an involvement of the cerebellum and the visual pathways after exposure to $\mathrm{Hg}$ vapour. These abnormalities of the central nervous system were not related to any of the indices of dose used in the present study, but were related to age and years since first exposure.

Another group of exposed subjects had reduced distal sensation, which was related both to cumulative $\mathrm{U}-\mathrm{Hg}$ and cumulative $\mathrm{A}-\mathrm{Hg}$. Some neurographical measurements also showed dose-response relations with the dose indices used in the present study. Current $\mathrm{U}-\mathrm{Hg}$ was associated with many neurographical measurements among the exposed subjects but not among the referents, which may indicate that $\mathrm{Hg}$ from remote exposure is still excreted and may have influence on the neurographical measurements.

We thank Tone Grande for her assistance in statistical matters and Ms Patricia Flor for her linguistic help. 
This study was carried out with financial support from Norsk Hydro A/S, Norway.

Requests for reprints to: Dag G Ellingsen, Department of Occupational Medicine, Telemark Central Hospital, N-3906 Porsgrunn, Norway.

1 Berlin M. Mercury. In: Friberg L, Nordberg GF, Vouk VB, eds. Handbook on the toxicology of metals. 2nd ed. Vol 2. Amsterdam: Elsevier, 1986;387-445.

2 Lille F, Hazemann P, Garnier R, Dally S. Effects of lead and mercury intoxications on evoked potentials. Clinical Toxicology 1988;26:103-16.

3 Levine SP, Cavender GD, Langolf GD, Albers JW. Elemental mercury exposure: peripheral neurotoxicity. $\mathrm{Br} F$ Ind Med 1982;39:136-9.

4 Vroom FQ, Greer M. Mercury vapour intoxication. Brain 1972;95:305-18.

5 Fawer RF, De Ribaupierre Y, Guillemin MP, Berode M, Lob $M$. Measurement of hand tremor induced by industrial exposure to metallic mercury. $\mathrm{Br}$ F Ind Med 1983;40:204-8.

6 Albers JW, Cavender GD, Levine SP, Langolf GD. Asymptomatic sensorimotor polyneuropathy in workers exposed to elemental mercury. Neurology 1982;32:1168-74.

7 Singer R, Valciukas JA, Rosenman KD. Peripheral neurotoxicity in workers exposed to inorganic mercury compounds. Arch Environ Health 1987;42:181-4.

8 Langauer-Lewowicka $\mathrm{H}$, Kazibutowska $\mathrm{Z}$. Multimodality evoked potentials in occupational exposure to metallic mercury vapour. Polish fournal of Occupational Medicine 1989;2:192-9.

9 Langolf GD, Chaffin DB, Henderson R, Whittle HP. Evaluation of workers exposed to elemental mercury using quantitative tests of tremor and neuromuscular functions. Am Ind Hyg Assoc F 1978;39:976-84.

10 Piikivi L, Tolonen U. EEG findings in chlor-alkali workers subjected to low long term exposure to mercury vapour. $\mathrm{Br} F$ Ind Med 1989;46:370-5.

11 Roels H, Abdeladim S, Braun M, Malchaire J, Lauwerys R. Detection of hand tremor in workers exposed to mercury vapor: a comparative study of three methods. Environ Res 1989;49:152-65.

12 Lamm O, Pratt H. Subclinical effects of exposure to inorganic mercury revealed by somatosensory-evoked potentials. Eur
Neurol 1985;24:237-43.

13 Chapman LJ, Sauter SL, Henning RA, Dodson VN, Reddan WG, Matthews CG. Differences in frequency of finger tremor in otherwise asymptomatic mercury workers. $\mathrm{Br} \mathrm{F}$ Ind Med 1990;47:838-43.

14 Smith RG, Vorwald AJ, Patil LS, Mooney TF. Effects of exposure to mercury in the manufacture of chlorine. Am Ind Hyg Assoc F 1970;31:687-700.

15 Ehrenberg RL, Vogt RL, Smith AB, et al. Effects of elemental mercury exposure at a thermometer plant. $A m$ f Ind Med 1991;19:495-507.

16 Shapiro IM, Cornblath DR, Sumner AJ, et al. Neurophysiological and neuropsychological function in mercury-exposed dentists. Lancet 1982;i:1 147-50.

17 Albers JW, Kallenbach LR, Fine LJ, et al. Neurological abnormalities associated with remote occupational elemental mercury exposure. Ann Neurol 1988;24:651-9.

18 He FS, Zhow XR, Lin BX, et al. Prognosis of mercury poisoning in mercury refinery workers. Ann Acad Med Singapore 1984;13:389-93.

19 Takahata N, Hayashi H, Watanabe S, Anso T. Accumulation of mercury in the brains of two autopsy cases with chronic inorganic mercury poisoning. Folia Psychiatrica et Neurologica Faponica 1970;24:60-9.

20 Hargreaves RJ, Evans JG, Janota I, Magos L, Cavanagh JB. Persistent mercury in nerve cells 16 years after metallic mercury poisoning. Neuropathol Appl Neurobiol 1988;14:443-52.

21 Kosta L, Byrne AR, Zelenko V. Correlation between selenium and mercury in man following exposure to inorganic mercury. Nature 1975;254:238-9.

22 Andersen A, Ellingsen DG, Mørland T, Kjuus H. A neurological and neurophysiological study of chloralkali workers previously exposed to mercury vapour. (Acta Neurol Scan) (in press).

23 Ellingsen DG, Holland RI, Thomassen Y, Ladro-Olstad M, Frech W, Kjuus $\mathrm{H}$. Mercury and selenium in workers previously exposed to mercury vapour at a chloralkali plant. $\mathrm{Br} \mathcal{F}$ Ind Med 1993;50:745-52.

24 Kleinbaum DG, Kupper LL, Muller KE. Applied regression analysis and other multivariable methods. 2nd ed. Boston: PWS-Kent, 1988.

25 Hursh JB, Greenwood MR, Clarkson TW, Allen J, Demuth S. The effect of ethanol on the fate of mercury vapor inhaled by man. F Pharmacol Exp Ther 1980;214:520-7.

26 Nylander M, Friberg L, Lind B. Mercury concentrations in the human brain and kidneys in relation to exposure from dental amalgam fillings. Swed Dent f 1987;11:179-87.

Accepted 26 October 1992 